\title{
STRONG WAVEFRONT LEMMA AND COUNTING LATTICE POINTS IN SECTORS
}

\author{
ALEXANDER GORODNIK, HEE OH AND NIMISH SHAH
}

Preliminary version as of October 29, 2018

\begin{abstract}
We compute the asymptotics of the number of integral quadratic forms with prescribed orthogonal decompositions and, more generally, the asymptotics of the number of lattice points lying in sectors of affine symmetric spaces. A new key ingredient in this article is the strong wavefront lemma, which shows that the generalized Cartan decomposition associated to a symmetric space is uniformly Lipschitz.
\end{abstract}

\section{INTRODUCTION}

One of the motivations of this paper is a certain counting problem in the space of quadratic forms. Let $\mathcal{S}_{W}$ be the vector space of all quadratic forms on a Euclidean space $W$ of dimension $d$. We fix an integral structure on $W$, and hence on $\mathcal{S}_{W}$. Let $\mathcal{Q}_{W}$ denote the subset of $\mathcal{S}_{W}$ consisting of quadratic forms of determinant \pm 1 , and set $\mathcal{Q}_{W}(\mathbb{Z})=\mathcal{Q}_{W} \cap \mathcal{S}_{W}(\mathbb{Z})$. Let $\|\cdot\|$ be any norm on $\mathcal{S}_{W}$. It follows from the main result of Duke, Rudnick and Sarnak [DRS], as well as, Eskin and McMullen [EM] that for $d \geq 3$ there exists a constant $c>0$ such that

$$
\#\left\{\boldsymbol{q} \in \mathcal{Q}_{W}(\mathbb{Z}):\|\boldsymbol{q}\|<T\right\} \sim_{T \rightarrow \infty} c \cdot T^{d(d-1) / 2} .
$$

Here we will consider a refinement of this problem that concerns counting quadratic forms with prescribed structure. Fix an orthogonal decomposition

$$
W=\oplus_{i=0}^{n} W_{i},
$$

and for $\Omega \subset \mathrm{SO}(W)$ and $\Omega^{\prime} \subset \mathcal{Q}_{W_{0}} \times \cdots \times \mathcal{Q}_{W_{n}}$, set

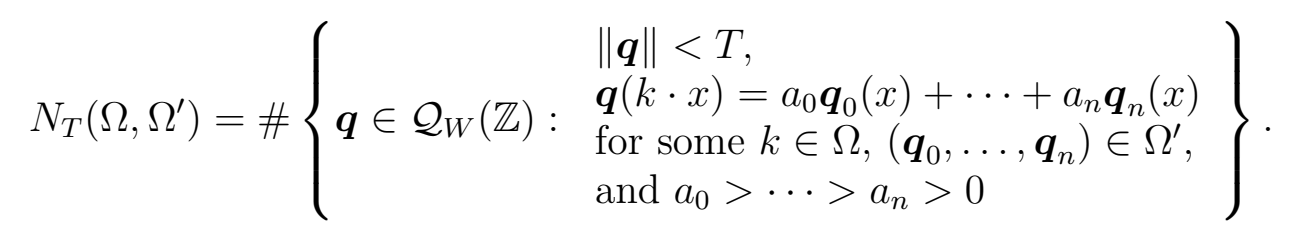

For example, if we choose $W_{i}$ 's to be one dimensional, then we are counting the number of quadratic forms in a ball of radius $T$ which can be diagonalized via conjugation by an element from a prescribed set $\Omega$ of orthogonal transformations

The first and the second authors partially supported by NSF 0400631 and NSF 0333397 respectively. 
to obtain a form with distinct eigenvalues in decreasing order of absolute values, and with prescribed sign $( \pm)$ in each diagonal entry.

Assuming that $\Omega$ and $\Omega^{\prime}$ are bounded measurable sets such that the subset $\Omega \Omega^{\prime}$ has positive measure and boundary of measure zerd 1 , we prove the following:

Theorem 1.4. For $d \geq 3$,

$$
N_{T}\left(\Omega, \Omega^{\prime}\right) \sim_{T \rightarrow \infty} c \cdot T^{d\left(d-\operatorname{dim} W_{n}\right) / 2}
$$

for some $c=c\left(\Omega \Omega^{\prime}\right)>0$.

Theorem 1.4 is an example of our general result (Theorem 1.13) on counting lattice points in sectors of affine symmetric spaces. In [DRS, EM] it is shown that the number of integral points in an affine symmetric $\mathbb{Q}$-variety in a sequence of growing subsets $S_{T}$ is asymptotic to the volume of $S_{T}$, provided the sets $S_{T}$ are well-rounded. A family of subsets $S_{T}$ being well-rounded means roughly that the volumes of neighborhoods of the boundaries of $S_{T}$ are uniformly negligible compared to the total volumes of $S_{T}$ (see (1.11) for the precise condition). In [DRS, EM], it is shown that the norm balls are well-rounded. However, in most situations, given a sequence of subsets $S_{T}$ which arises naturally in the geometric or number-theoretic contexts in the category of affine symmetric spaces, it is highly non-trivial to determine whether the family $S_{T}$ is well-rounded.

The main result of this paper is to show that sectors in affine symmetric spaces define a well-rounded family of growing subsets, and consequently, we obtain the asymptotic counting of lattice points in sectors. The main technical lemma needed is what we call 'strong wave front lemma', a terminology reflecting it being a stronger version of the wavefront lemma introduced by Eskin and McMullen [EM].

Now we introduce notation that we use throughout the paper. Let $G$ be a connected noncompact semisimple Lie group with finite center. A closed subgroup $H$ of $G$ is called symmetric if its identity component coincides the the identity component of the set of fixed points of an involution, say $\sigma$, of $G$. In this case, the homogeneous space $G / H$ is called an affine symmetric space. Recall that a maximal compact subgroup of $G$ is a symmetric subgroup associated to a Cartan involution on $G$. Affine symmetric spaces have many features similar to Riemannian symmetric spaces. In particular, a generalized Cartan decomposition holds:

$$
G=K A H
$$

where $K$ is a maximal compact subgroup of $G$ compatible with $H$, and $A$ is a Cartan subgroup corresponding to the pair $(K, H)$.

More precisely, there exists a Cartan involution $\theta$ of $G$ which commutes with $\sigma$, and let $K=\{g \in G: \theta(g)=g\}$, which is a maximal compact subgroup of $G$.

\footnotetext{
${ }^{1}$ The measure of $\Omega \Omega^{\prime}$ is understood in terms of the identification (3.2) and (3.3).
} 
Let $\mathfrak{g}, \mathfrak{h}$, and $\mathfrak{k}$ denote the Lie algebras associated to $G, H$ and $K$, respectively. Let $\theta$ and $\sigma$ also denote their differentials on $\mathfrak{g}$. Since $H$ and $K$ are $\theta$ stable, we have the following orthogonal decomposition with respect to the killing form on $\mathfrak{g}: \mathfrak{g}=\mathfrak{k} \oplus \mathfrak{p}$, and $\mathfrak{g}=\mathfrak{h} \oplus \mathfrak{q}$, where $\mathfrak{p}$ and $\mathfrak{q}$ are the $(-1)$-eigenspaces of $\theta$ and $\sigma$, respectively. Let $\mathfrak{a}$ denote the maximal abelian subalgebra of $\mathfrak{p} \cap \mathfrak{q}$ which can be extended to a maximal abelian subalgebra, say $\mathfrak{b}$, of $\mathfrak{p}$. Let $A$ denote the analytic subgroup of $G$ associated to $\mathfrak{a}$. This $A$ is called the Cartan subgroup corresponding to the symmetric pair $(K, H)$.

Wavefront Lemma (Eskin and McMullen [EM]). Given any neighborhood $\mathcal{O}$ of $e$ in $G$, there exists a neighborhood $\tilde{\mathcal{O}}$ of $e$ in $G$ such that

$$
\tilde{\mathcal{O}} g \subset g \mathcal{O} H, \quad \forall g \in K A .
$$

Next we will strengthen this result for uniformly regular elements of $g \in G$. For this we will need additional notation (cf. [Sc, Ch. 7], [HS, Part II] or [GOS]). Let $\mathfrak{g}^{\alpha}$ denote a simultaneous eigenspace for ad $\mathfrak{a}$ action on $\mathfrak{g}$ associated to the linear character $\alpha \in \mathfrak{a}^{*}$. Let $\Sigma_{\sigma}=\left\{\alpha \in \mathfrak{a}^{*}: \mathfrak{g}^{*} \neq 0\right\}$. Then $\mathfrak{g}=\sum_{\alpha \in \Sigma_{\sigma} \cup\{0\}} \mathfrak{g}^{\alpha}$, and $\Sigma_{\sigma}$ forms a root system. Choose a closed positive Weyl chamber $A^{+} \subset A$. Let $\Sigma_{\sigma}^{+}$denote the set of positive roots and $\Delta_{\sigma}$ the corresponding system of positive simple roots. The associated Weyl group is given by $\mathcal{W}_{\sigma}=N_{K}(\mathfrak{a}) / Z_{K}(\mathfrak{a})$. One can choose a set $\mathcal{W} \subset N_{K}(\mathfrak{a}) \cap N_{K}(\mathfrak{b})$ of coset representatives of $N_{K}(\mathfrak{a}) / N_{K \cap H}(\mathfrak{a}) Z_{K}(\mathfrak{a})$. Then

$$
G=\cup_{w \in \mathcal{W}} K A^{+} w H .
$$

For any $c>0$, an element $g=k a w h \in K A^{+} \mathcal{W} H$ will be called $c$-regular if $\alpha(\log a) \geq c$ for all $\alpha \in \Delta_{\sigma}$; (here and later, our notation indicates that $k \in K$, $a \in A^{+}, w \in \mathcal{W}$, and $\left.h \in H\right)$. Otherwise, we call such an element $c$-singular.

We fix a Riemannian metric on $G$ and denote by $\mathcal{O}_{\varepsilon}$ the $\varepsilon$-ball at identity.

Theorem 1.6 (Strong wavefront lemma-I). Given $c>0$, there exist $\ell>1$ and $\varepsilon_{0}>0$ such that for every c-regular $g=k a w h \in K A^{+} w H$ and $0<\varepsilon<\varepsilon_{0}$,

$$
\mathcal{O}_{\varepsilon} \cdot g \subset\left(K \cap \mathcal{O}_{\ell \varepsilon}\right) k \cdot\left(A \cap \mathcal{O}_{\ell \varepsilon}\right) a \cdot w\left(H \cap \mathcal{O}_{\ell \varepsilon}\right) h .
$$

The continuity of the Cartan decomposition for Riemannian symmetric spaces (that is, when $H=K$ ) was independently shown in Nevo [N, Proposition 7.3] and by Gorodnik and Oh [GO, Theorem 2.1]. While the proof of [N] uses embeddings of $G$ in linear groups, the proof of [GO] is based on geometric properties of the Riemannian symmetric spaces. The strong wavefront lemma was used in [N] to prove maximal inequalities for cube averages on semisimple groups and in [GO] to compute the asymptotics of the number of lattice points lying in sectors.

Theorem 1.6 fails on the set of singular elements; for example, in $\mathrm{SL}_{2}(\mathbb{R})$ if $\Omega$ is a small neighborhood of the $e$, then $(\Omega \cap K)(\Omega \cap A)(\Omega \cap K)$ does not contain 
a neighborhood of the $e$ in $\mathrm{SL}_{2}(\mathbb{R})$. To state a version of the strong wavefront lemma that holds for singular elements, we introduce additional notation. Given $J \subset \Delta_{\sigma}$, an element $k a w h \in K A^{+} \mathcal{W} H$ is called $(J, c)$-regular if $\alpha(\log a) \geq c$ for all $\alpha \in J$. Let $I=\Delta_{\sigma} \backslash J$. We set $A_{I}=\exp (\operatorname{ker} I) \subset A$. Let $M_{I}$ be the analytic semisimple subgroup whose Lie algebra is generated by $\mathfrak{g}^{ \pm \beta}, \beta \in \Sigma_{\sigma}^{+} \cap\langle I\rangle$. Then $M_{I}$ centralizes $A_{I}$. Now

$$
G=\cup_{w \in \mathcal{W}} K M_{I} A_{I}^{+} w H \quad \text { and } \quad M_{I} \cap A_{I}=\{e\},
$$

where $A_{I}^{+}=A_{I} \cap A^{+}$.

Theorem 1.7 (Strong wavefront lemma-II). Given $c>0$, there exist $\ell>1$ and $\varepsilon_{0}>0$ such that for any $I \subset \Delta_{\sigma}$ and $J=\Delta_{\sigma} \backslash I$, and every $g=k a w h \in K A^{+} \mathcal{W} H$ and $0<\varepsilon<\varepsilon_{0}$, if $g$ is $(J, c)$-regular, then

$$
\mathcal{O}_{\varepsilon} \cdot g \subset\left(K \cap \mathcal{O}_{\ell \varepsilon}\right) k \cdot\left(M_{I} \cap \mathcal{O}_{\ell \varepsilon}\right) \cdot\left(A_{I} \cap \mathcal{O}_{\ell \varepsilon}\right) a \cdot w\left(H \cap \mathcal{O}_{\ell \varepsilon}\right) h .
$$

Remark 1.8. Observe that by [GOS, Corollary 4.7], since $w v_{0}$ is fixed by the symmetric subgroup $M_{I} \cap w H w^{-1}$ of $M_{I}$, the orbit $M_{I}\left(w v_{0}\right)$ is closed. Since $M_{I} \subset Z_{G}\left(A_{I}\right)$, we have $M_{I} a w v_{0}=a M_{I} w v_{0}$ is closed. Thus, the set $K M_{I} a w v_{0}$ is closed for any $a \in A_{I}$. Moreover the natural map $K M_{I} /\left(M_{I} \cap w H w^{-1}\right) \rightarrow$ $K M_{I} a w v_{0}$ given by $k m\left(M_{I} \cap w H w^{-1}\right) \mapsto k m a w v_{0}$ is a homeomorphism.

A natural generalization of the Cartan decomposition for Riemannian symmetric spaces is the decomposition

$$
G=K \tilde{A}^{+} H
$$

where $\tilde{A^{+}}$is a Weyl chamber in $A$ with respect to the Weyl group $\left(N_{G}(A) \cap\right.$ $K \cap H) /\left(Z_{G}(A) \cap K \cap H\right)$. In Section 4, we will obtain the strong wavefront lemmas with respect to the decomposition (1.9), which generalize Theorem 1.6 and Theorem 1.7 .

Well-roundedness of sectors. Let $\iota: G \rightarrow \mathrm{GL}(W)$ be an irreducible representation of $G$ and $v_{0} \in W$ such that if $H$ denotes the stabilizer of $v_{0}$ then $H$ is a symmetric subgroup of $G$. Therefore by [GOS, Corollary 4.7] the orbit $V=G v_{0}$ closed. Hence it can be realized as an affine symmetric space $G / H$. Let $\Gamma$ be a lattice in $G$. We suppose that $H \cap \Gamma$ is also a lattice in $H$. In particular, $H \Gamma$ is closed in $G$ (, and hence $\Gamma v_{0}$ is a discrete subset of $W$. For a norm $\|\cdot\|$ on $W$, we set

$$
B_{T}=\{w \in W:\|w\|<T\} .
$$

It was shown in DRS, EM that the orbit $\Gamma v_{0}$ is "equidistributed" with respect to the sets $V \cap B_{T}$ in the following sense:

$$
\#\left(\Gamma v_{0} \cap B_{T}\right) \sim_{T \rightarrow \infty} \operatorname{Vol}\left(V \cap B_{T}\right)
$$

where $\mathrm{Vol}$ is the $G$-invariant measure on $V \cong G / H$ determined by the Haar measures on $G$ and $H$ chosen such that $\operatorname{Vol}(G / G \cap \Gamma)=\operatorname{Vol}(H / H \cap \Gamma)=1$. In 
fact, it was shown in EM that (1.10) holds for any well-rounded family of sets $S_{T} \subset V$ in place of $V \cap B_{T}$. Recall that a family $\left\{S_{T}\right\}$ is called well-rounded if for any $\varepsilon>0$ there exists a neighborhood $\mathcal{O}$ of $e$ in $G$ such that

$$
\frac{\operatorname{Vol}\left(\mathcal{O} \cdot \partial S_{T}\right)}{\operatorname{Vol}\left(S_{T}\right)}<\varepsilon
$$

for all sufficiently large $T>0$. For any $I \subset \Delta_{\sigma}, w \in \mathcal{W}$ and $\Omega \subset K M_{I} /\left(M_{I} \cap\right.$ $\left.w H w^{-1}\right)$, we consider a family of sets

$$
S_{T}(\Omega, w)=\tilde{\Omega} A_{I}^{+} w v_{0} \cap B_{T},
$$

where $\tilde{\Omega} \subset K M_{I}$ is such that $\Omega=\tilde{\Omega}\left(M_{I} \cap w H w^{-1}\right)$; the set $S_{T}(\Omega, w)$ is well defined because $m a w v_{0}=a w v_{0}$ for all $a \in A_{I}$ and $m \in\left(M_{I} \cap w H w^{-1}\right)$.

Using the strong wavefront lemma, and the volume computation in GOS (cf. Proposition 3.8) we obtain the following:

Theorem 1.13. For every $I \subset \Delta_{\sigma}, w \in \mathcal{W}$, and a bounded measurable set $\Omega \subset K M_{I} /\left(M_{I} \cap w H w^{-1}\right)$ with positive measure and boundary of measure zerd2, the family $\left\{S_{T}(\Omega, w)\right\}_{T \rightarrow \infty}$ is well-rounded. In particular,

$$
\#\left(\Gamma v_{0} \cap S_{T}(\Omega, w)\right) \sim_{T \rightarrow \infty} \operatorname{Vol}\left(S_{T}(\Omega, w)\right) \sim_{T \rightarrow \infty} C_{I}(\Omega, w) \cdot T^{a_{I}}(\log T)^{b_{I}-1},
$$

where $a_{I} \in \mathbb{Q}^{+}, b_{I} \in \mathbb{N}$, and $C_{I}(\Omega, w)>0$.

We will give explicit formulas for $a_{I}, b_{I}$, and $C_{I}(\Omega, w)$ in section 3.2. In particular, $C_{I}(\Omega, w)$ can be computed using a $G$-invariant measure supported on one of the components of the Satake boundary of $V$.

Remark 1.14. (1) Although a similar counting question was considered in [GOS], the sets $S_{T}(\Omega, w)$ do not fit into the framework of [GOS]. For the space of quadratic forms $\mathcal{Q}_{W}$, the counting results in GOS are always of order $T^{(\operatorname{dim} W)(\operatorname{dim} W-1) / 2}$ (see [GOS, Section 2.3]). On the other hand, Theorem 1.4 exhibits different asymptotic behaviors depending on the choice of the decomposition (1.2).

(2) In order to deduce Theorem 1.13 from Theorem 1.7, which applies only to $(J, c)$-regular elements, we show that the set of non- $(J, c)$-regular elements in $S_{T}(\Omega, w)$ has negligible volume compared to the volume of $S_{T}(\Omega, w)$ for sufficiently small values of $c$.

1.1. Acknowledgment. We would like to thank Yves Benoist for useful comments.

\footnotetext{
${ }^{2}$ The measure on $K M_{I} /\left(M_{I} \cap w H w^{-1}\right)$ is understood in terms of the identification (3.2) and (3.3).
} 


\section{Strong WAVEFront LEMma}

This section is devoted to the proofs of Theorems 1.6 and 1.7. We use the same notation as in the introduction. Since any two Riemannian metrics are biLipschitz in a neighborhood of identity, it suffices to prove the theorems for one such metric. It will be convenient to work with the right-invariant Riemannian metric $d$ induced by the positive definite form

$$
B(X, Y)=-\operatorname{Tr}(\operatorname{ad} X \circ \operatorname{ad}(\theta(Y)), \quad X, Y \in \mathfrak{g} .
$$

We will use the following properties of $B$ :

$$
\begin{aligned}
& B\left(\mathfrak{g}_{\alpha}, \mathfrak{g}_{\beta}\right)=0 \quad \text { for all } \alpha \neq \beta \in \Sigma_{\sigma} \cup\{0\}, \\
& B^{\theta}=B^{\sigma}=B .
\end{aligned}
$$

Remark 2.1. In many of the results stated in the introduction, we fix $w \in \mathcal{W}$ representing a Weyl group element. The explanation given below shows that for proofs, we can assume that $w=e$ and have simpler notation.

Let $i_{w}$ denote the inner conjugation on $G$ by $w$; that is, $i_{w}(g)=w g w^{-1}$ for all $g \in G$. Then $\sigma_{w}:=i_{w} \circ \sigma \circ i_{w}^{-1}$ is also an involution of $G$ and $w H w^{-1}$ is the associated symmetric subgroup. Note that $\sigma_{w}(a)=a^{-1}$ for any $a \in A$. Also $\theta \circ \sigma_{w}=\sigma_{w} \circ \theta$. Therefore in order to prove some of the results stated in the introduction for a fixed $w \in \mathcal{W}$, we can replace $\sigma$ by $\sigma_{w}, H$ by $w H w^{-1}$, and $v_{0}$ by $w v_{0}$, and assume that $w=e$.

For $\varepsilon>0$ and $S \subset G$, we set

$$
S_{\varepsilon}=\{s \in S: d(s, e)<\varepsilon\} .
$$

For $I \subset \Delta_{\sigma}$ and $c>0$, we define

$$
A_{I}^{+}(c)=\left\{a \in A^{+}: \beta(\log a) \geq c \text { if } \beta \in \Delta_{\sigma}-I \text { and } \beta(\log a)<c \text { if } \beta \in I\right\} .
$$

For instance, if $I=\{\beta\}$, then $A_{I}^{+}(c)$ forms a system of neighborhoods of the wall $\left\{a \in A^{+}: \beta(\log a)=0\right\}$ in $A^{+}$.

Theorem 2.2. For $I \subset \Delta_{\sigma}$ and $c>0$, there exist $\varepsilon_{0}>0$ and $\sigma>1$ such that for every $0<\varepsilon<\varepsilon_{0}$ and $a \in A_{I}^{+}(c)$,

$$
G_{\varepsilon} \cdot a \subset K_{\sigma \varepsilon} \cdot Z_{I, \sigma \varepsilon} \cdot a \cdot H_{\sigma \varepsilon} .
$$

We consider the Lie subalgebra

$$
\mathfrak{n}_{I}^{+}=\bigoplus_{\beta \in \Sigma_{\sigma}^{+}:\left.\beta\right|_{\mathfrak{a}_{I}} \neq 0} \mathfrak{g}_{\beta} \quad \text { and } \quad \mathfrak{n}_{I}^{-}=\bigoplus_{\beta \in \Sigma_{\sigma}^{+}:\left.\beta\right|_{\mathfrak{a}_{I}} \neq 0} \mathfrak{g}_{-\beta},
$$

and the corresponding analytic subgroups $N_{I}^{+}$and $N_{I}^{-}$. Note that the Lie algebra of $Z_{I}$ is given by

$$
\mathfrak{z}_{I}=\bigoplus_{\beta \in \Sigma_{\sigma} \cup\{0\}:,\left.\beta\right|_{\mathfrak{a}_{I}}=0} \mathfrak{g}_{\beta},
$$


and we have the decomposition

$$
\mathfrak{g}=\mathfrak{n}_{I}^{-} \oplus \mathfrak{z}_{I} \oplus \mathfrak{n}_{I}^{+} .
$$

Lemma 2.4. There exist $\theta>1$ and $\varepsilon_{0}>0$ such that for every $0<\varepsilon<\varepsilon_{0}$,

$$
G_{\varepsilon} \subset N_{I, \theta \varepsilon}^{-} Z_{I, \theta \varepsilon} H_{\theta \varepsilon} \quad \text { and } \quad G_{\varepsilon} \subset K_{\theta \varepsilon} Z_{I, \theta \varepsilon} N_{I, \theta \varepsilon}^{+} .
$$

Proof. Since $\left.\sigma\right|_{\mathfrak{a}}=-i d$, we have $\sigma\left(\mathfrak{n}_{I}^{-}\right) \subset \mathfrak{n}_{I}^{+}$, and for every $x \in \mathfrak{n}_{I}^{+}$,

$$
x=(x+\sigma(x))-\sigma(x) \in \mathfrak{h}+\mathfrak{n}_{I}^{-} .
$$

Hence, it follows from (2.3) that

$$
\mathfrak{g}=\mathfrak{n}_{I}^{-}+\mathfrak{z}_{I}+\mathfrak{h} .
$$

Since $\mathfrak{n}_{I}^{-} \cap \mathfrak{h}=0$, there exists a subspace $\mathfrak{z}_{0}$ of $\mathfrak{z}_{I}$ such that

$$
\mathfrak{g}=\mathfrak{n}_{I}^{-} \oplus \mathfrak{z}_{0} \oplus \mathfrak{h} .
$$

Then the product map $N_{I}^{-} \times \exp \left(\mathfrak{z}_{0}\right) \times H \rightarrow G$ is a diffeomorphism at a neighborhood of the identity. In particular, it is bi-Lipschitz, and the first claim follows. The proof of the second claim is similar.

Lemma 2.5. For $I \subset \Delta_{\sigma}$ and $c>0$, there exist $\varepsilon_{0}>0$ and $\alpha \in(0,1)$ such that for every $0<\varepsilon<\varepsilon_{0}$ and $a \in A_{I}^{+}(c)$,

$$
a^{-1} N_{I, \varepsilon}^{+} a \subset N_{I, \alpha \varepsilon}^{+} \quad \text { and } \quad a N_{I, \varepsilon}^{-} a^{-1} \subset N_{I, \alpha \varepsilon}^{-} .
$$

Proof. For

$$
X=\sum_{\beta \in \Sigma_{\sigma}^{+},\left.\beta\right|_{\mathfrak{a}_{I}} \neq 0} X_{\beta} \in \mathfrak{n}_{I}^{+}, \quad X_{\beta} \in \mathfrak{g}_{\beta}
$$

we have

$$
\operatorname{Ad}\left(a^{-1}\right) X=\sum_{\beta} \operatorname{Ad}\left(a^{-1}\right) X_{\beta}=\sum_{\beta} e^{-\beta(\log a)} X_{\beta} .
$$

Note that if $\beta=\sum_{\alpha \in \Delta_{\sigma}} n_{\alpha} \alpha \in \Sigma_{\sigma}^{+}$with $n_{\alpha} \geq 0$ satisfies $\left.\beta\right|_{\mathfrak{a}_{I}} \neq 0$, then $n_{\alpha} \geq 1$ for some $\alpha \in \Delta_{\sigma}-I$. Hence, for $a \in A_{I}^{+}(c)$, we have $\beta(\log a) \geq c$ and

$$
\left\|\operatorname{Ad}\left(a^{-1}\right) X_{\beta}\right\| \leq e^{-c}\left\|X_{\beta}\right\| \text {. }
$$

Since the root spaces $\mathfrak{g}_{\beta}$ are orthogonal to each other,

$$
\left\|\operatorname{Ad}\left(a^{-1}\right) X\right\| \leq e^{-c}\|X\| \text {. }
$$

Since the differential of the exponential map exp $: \mathfrak{n}_{I}^{+} \rightarrow N_{I}^{+}$is identity at 0 , we can find a small ball $U$ at 0 in $\mathfrak{n}_{I}^{+}$such that for every $Y \in U$.

$$
e^{-c / 3}\|Y\| \leq d(\exp (Y), e) \leq e^{c / 3}\|Y\| .
$$


Note that for $a \in A^{+}$, we have $\operatorname{Ad}\left(a^{-1}\right) U \subset U$. Combining (2.6) and (2.7), we deduce that for $a \in A_{I}^{+}(c)$ and $n=\exp (X) \in \exp (U)$,

$$
\begin{aligned}
d\left(a^{-1} n a, e\right) & =d\left(\exp \left(\operatorname{Ad}\left(a^{-1}\right) X\right), e\right) \leq e^{c / 3}\left\|\operatorname{Ad}\left(a^{-1}\right) X\right\| \\
& \leq e^{-2 c / 3}\|X\| \leq e^{-c / 3} d(n, e) .
\end{aligned}
$$

This proves the claim for $N_{I}^{+}$. The claim for $N_{I}^{-}$is proved similarly.

Lemma 2.8. For $I \subset \Delta_{\sigma}$ and $\tau>1$, there exists $\varepsilon_{0}>0$ such that for every $z \in Z_{I, \varepsilon_{0}}$ and $0<\varepsilon<\varepsilon_{0}$,

$$
z N_{\varepsilon}^{+} z^{-1} \subset N_{\tau \varepsilon}^{+} \quad \text { and } \quad z N_{\varepsilon}^{-} z^{-1} \subset N_{\tau \varepsilon}^{-} .
$$

Proof. It is easy to check that $L_{I}$ normalizes $N_{I}^{ \pm}$.

We can choose $\varepsilon_{0}>0$ so that

$$
\begin{gathered}
\|\operatorname{Ad}(z) X\| \leq \tau^{1 / 3}\|X\|, \\
\tau^{-1 / 3}\|X\| \leq d(\exp (X), e) \leq \tau^{1 / 3}\|X\|, \quad X \in Z_{I, \varepsilon_{0}}, X \in \mathfrak{n}_{I}^{+}, \\
\operatorname{Ad}\left(Z_{I, \varepsilon_{0}}\right) \exp ^{-1}\left(N_{I, \varepsilon_{0}}^{+}\right) .
\end{gathered}
$$

Then for every $n=\exp (X) \in N_{I, \varepsilon_{0}}^{+}$,

$$
\begin{aligned}
d\left(z n z^{-1}, e\right) & =d(\exp (\operatorname{Ad}(z) X), e) \leq \tau^{1 / 3}\|\operatorname{Ad}(z) X\| \\
& \leq \tau^{2 / 3}\|X\| \leq \tau d(n, e) .
\end{aligned}
$$

This proves the first part of the lemma. The proof of the second part is similar.

Lemma 2.9. For $I \subset \Delta_{\sigma}$ and $\gamma>1$, there exists $\varepsilon_{0}>0$ such that for every $0<\varepsilon<\varepsilon_{0}$,

$$
N_{I, \varepsilon}^{+} \subset N_{I, \gamma \varepsilon}^{-} Z_{I, \varepsilon} H_{2 \gamma \varepsilon} \quad \text { and } \quad N_{I, \varepsilon}^{-} \subset K_{2 \gamma \varepsilon} Z_{I, \varepsilon} N_{I, \gamma \varepsilon}^{+} \text {. }
$$

Proof. As in the proof of Lemma 2.4, we choose a subspace $\mathfrak{z}_{0}$ of $\mathfrak{z}_{I}$ such that the product map $N_{I}^{-} \times \exp \left(\mathfrak{z}_{0}\right) \times H \rightarrow G$ is a diffeomorphism in a neighborhood of the identity. Denote by $f$ the local inverse the product map:

$$
f=\left(f_{1}, f_{2}, f_{3}\right): U \rightarrow N_{I}^{-} \times \exp \left(\mathfrak{z}_{0}\right) \times H
$$

where $U$ is a neighborhood of identity in $G$. For $X \in \mathfrak{n}_{I}^{+}$, the derivative $(d f)_{e}$ is given by

$$
(d f)_{e}(X)=(-\sigma(X), 0, X+\sigma(X)) \in \mathfrak{n}_{I}^{-} \oplus \mathfrak{z}_{0} \oplus \mathfrak{h} .
$$

Since the Riemannian metric at identity is invariant under $\sigma$, we have for $X \in \mathfrak{n}_{I}^{+}$,

$$
\left\|\left(d f_{1}\right)_{e}(X)\right\|=\|X\|, \quad\left(d f_{2}\right)_{e}=0, \quad\left\|\left(d f_{3}\right)_{e}(X)\right\| \leq 2\|X\| .
$$

This implies that for sufficiently small $\varepsilon>0$,

$$
f\left(N_{I, \varepsilon}^{+}\right) \subset N_{I, \gamma \varepsilon}^{-} \times Z_{I, \varepsilon} \times H_{2 \gamma \varepsilon} .
$$

This proves the first claim. The proof of the second claim is similar. 
Lemma 2.10. For $I \subset \Delta_{\sigma}$ and $c>0$, there exist $0<\beta<1$ and $\varepsilon_{0}>0$ such that for every $0<\varepsilon, \delta<\varepsilon_{0}$ and $a \in A_{I}^{+}(c)$,

$$
K_{\varepsilon} Z_{I, \varepsilon} a Z_{I, \varepsilon} N_{I, \delta}^{+} H_{\varepsilon} \subset K_{\varepsilon+4 \delta} Z_{I, \varepsilon+4 \delta} a Z_{I, \varepsilon+4 \delta} N_{I, \beta \delta}^{+} H_{\varepsilon+4 \delta} .
$$

Proof. For simplicity, we write $N_{I}^{ \pm}=N^{ \pm}$and $Z_{I}=Z$.

Choose $\alpha=\alpha(c) \in(0,1)$ as in Lemma 2.5, $\gamma \in(1,2)$ so that $\alpha \gamma^{2}<1$, and $\tau>1$ so that $\tau^{5} \alpha \gamma^{2}<1$. Let $\varepsilon_{0}>0$ be such that Lemma 2.5, Lemma 2.8, and Lemma 2.9 hold. Fixing $0<\varepsilon<\varepsilon_{0}$, let $k_{0} \in K_{\varepsilon}, x_{0}, y_{0} \in Z_{\varepsilon}, n_{0}^{+} \in N_{\delta}^{+}$, and $h_{0} \in H_{\varepsilon}$. Then

$$
\begin{aligned}
& k_{0} x_{0} a y_{0} n_{0}^{+} h_{0} \\
& =k_{0} x_{0} a y_{0}\left(n_{1}^{-} y_{1} h_{1}\right) h_{0} \\
& =k_{0} n_{2}^{-} x_{0} a y_{0} y_{1} h_{1} h_{0} \\
& =k_{0}\left(k_{2} x_{2} n_{2}^{+}\right) x_{0} a y_{0} y_{1} h_{1} h_{0} \\
& \text { with } k_{2} \in K_{2 \tau^{2} \alpha \gamma^{2} \delta}, x_{2} \in Z_{\tau^{2} \alpha \gamma \delta}, n_{2}^{+} \in N_{\tau^{2} \alpha \gamma^{2} \delta}^{+} \\
& =k_{0} k_{2}\left(x_{2} x_{0} a y_{0} y_{1}\right) n_{3}^{+} h_{1} h_{0} \\
& \text { by Lemma 2.9 } \\
& \text { with } n_{1}^{-} \in N_{\gamma \delta}^{-}, y_{1} \in Z_{\delta}, h_{1} \in H_{2 \gamma \delta} \\
& \text { by Lemma } 2.8 \text { and Lemma } 2.5 \\
& \text { with } n_{2}^{-} \in N_{\tau^{2} \alpha \gamma \delta}^{-} \\
& \text {by Lemma 2.9, }
\end{aligned}
$$

Since $\tau^{5} \alpha \gamma^{2}<1$, we have

$$
k_{0} k_{2} \in K_{\varepsilon+4 \delta}, \quad x_{2} x_{0}, y_{0} y_{1} \in Z_{\varepsilon+4 \delta}, \quad n_{3}^{+} \in N_{\beta \delta}^{+}, \quad h_{1} h_{0} \in H_{\varepsilon+4 \delta} .
$$

where $\beta=\tau^{5} \alpha^{2} \gamma^{2}<1$.

Proof of Theorem 2.2. Set $N_{I}^{ \pm}=N^{ \pm}$and $Z_{I}=Z$ for simplicity. In view of Remark 2.1 without loss of generality we may assume that $w=e$.

We choose $\varepsilon_{0}>0$ so that Lemma 2.4 (for some $\theta>1$ ), Lemma 2.5, and Lemma 2.10 hold. Because of Lemma 2.4, it suffices to show that

$$
K_{\varepsilon} Z_{\varepsilon} N_{\varepsilon}^{+} \cdot a \subset K_{\sigma \varepsilon}\left(Z_{\sigma \varepsilon} a\right) H_{\sigma \varepsilon}
$$

for some $\sigma>1$. Also by Lemma 2.5,

$$
K_{\varepsilon} Z_{\varepsilon} N_{\varepsilon}^{+} \cdot a \subset K_{\varepsilon}\left(Z_{\varepsilon} a Z_{\varepsilon}\right) N_{\varepsilon}^{+} H_{\varepsilon} .
$$

Now we can apply Lemma 2.10 inductively. We consider $\varepsilon>0$ such that

$$
\varepsilon+\frac{4 \varepsilon}{1-\beta}<\varepsilon_{0} .
$$

Setting $\varepsilon_{0}=\delta_{0}=\varepsilon$, we apply Lemma 2.10 to find

$$
\varepsilon_{i+1}<\varepsilon_{i}+2 \delta_{i}, \quad \delta_{i+1}<\beta \delta_{i}
$$


such that for every $a \in A_{I}^{+}(c)$,

$$
K_{\varepsilon_{i}} Z_{\varepsilon_{i}} a Z_{\varepsilon_{i}} N_{\delta_{i}}^{+} H_{\varepsilon_{i}} \subset K_{\varepsilon_{i+1}} Z_{\varepsilon_{i+1}} a Z_{\varepsilon_{i+1}} N_{\delta_{i+1}}^{+} H_{\varepsilon_{i+1}} .
$$

Note that

$$
\delta_{i}<\varepsilon \beta^{i} \quad \text { and } \quad \varepsilon_{i}<\varepsilon+4 \varepsilon \frac{1-\beta^{i}}{1-\beta} .
$$

Hence by (2.11), $\varepsilon_{i}, \delta_{i}<\varepsilon_{0}$, and we can continue this process indefinitely.

It follows that for every $g \in K_{\varepsilon}\left(Z_{\varepsilon} a Z_{\varepsilon}\right) N_{\varepsilon}^{+} H_{\varepsilon}$, there exist sequences $k_{i} \in$ $K_{\varepsilon_{i}}, x_{i}, y_{i} \in Z_{\varepsilon_{i}}, n_{i} \in N_{\delta_{i}}^{+}, h_{i} \in H_{\varepsilon_{i}}$ such that $g=k_{i} x_{i} a y_{i} n_{i} h_{i}$ for all $i \geq 1$. Since $\delta_{i} \rightarrow 0, n_{i} \rightarrow e$. Also, passing to a subsequence, we may assume that $k_{i} \rightarrow k, x_{i} \rightarrow x, y_{i} \rightarrow y, h_{i} \in h$. Then

$$
g=k x a y h \subset K_{\rho \varepsilon} Z_{\rho \varepsilon} a Z_{\rho \varepsilon} H_{\rho \varepsilon}
$$

with $\rho=1+4(1-\beta)^{-1}$. We have decomposition $a=a_{1} a_{2}$ where $a_{1} \in A_{I}^{+}$and $a_{2}$ is in the fixed compact set determined by $c$. This implies that for some $\tau>1$,

$$
a Z_{\rho \varepsilon} a^{-1} \subset Z_{\tau \rho \varepsilon}
$$

and the theorem follows.

Proof of Theorem 1.7. There exists $\zeta>1$ such that $k^{-1} \mathcal{O}_{\varepsilon} k \subset \mathcal{O}_{\zeta \varepsilon}$ for every $k \in K$. Then for $g=k a w h \in K A^{+} \mathcal{W} H$, we have

$$
\mathcal{O}_{\varepsilon} \cdot g \subset k\left(\mathcal{O}_{\zeta \varepsilon} a\right) w h
$$

Due to Remark 2.1, without loss of generality, we may assume that $w=e$.

Since $M_{I_{1}} \subset M_{I_{2}}$ for $I_{1} \subset I_{2}$, we may assume that $J$ is maximal such that $a$ is $(J, c)$-regular. Then $a \in A_{I}^{+}(c)$. We have the decomposition

$$
\mathfrak{z}_{I}=\left(\mathfrak{z}_{I} \cap \mathfrak{k}\right) \oplus\left(\mathfrak{m}_{I} \cap \mathfrak{p} \cap \mathfrak{q}\right) \oplus \mathfrak{a}_{I} \oplus\left(\mathfrak{z}_{I} \cap \mathfrak{h}\right)
$$

(see [GOS, equation (4.24)]). Hence, the product map

$$
\left(Z_{I} \cap K\right) \times \exp \left(\mathfrak{m}_{I} \cap \mathfrak{p} \cap \mathfrak{q}\right) \times A_{I} \times\left(Z_{I} \cap H\right) \rightarrow Z_{I}
$$

is a diffeomorphism in a neighborhood of identity, and there exists $\eta>1$ such that for sufficiently small $\varepsilon>0$,

$$
Z_{I, \varepsilon} \subset\left(Z_{I} \cap K\right)_{\eta \varepsilon} \exp \left(\mathfrak{m}_{I} \cap \mathfrak{p} \cap \mathfrak{q}\right)_{\eta \varepsilon} A_{I, \eta \varepsilon}\left(Z_{I} \cap H\right)_{\eta \varepsilon} .
$$

Therefore, it follows from Theorem 2.2 that

$$
\mathcal{O}_{\varepsilon} \cdot a \subset K_{\sigma \varepsilon} Z_{\sigma \varepsilon} a H_{\sigma \varepsilon} \subset K_{(\sigma+\sigma \eta) \varepsilon} M_{I, \sigma \eta \varepsilon}\left(A_{I, \sigma \eta \varepsilon} a\right) H_{(\sigma+\sigma \eta) \varepsilon} .
$$

This proves the theorem. 
Proof of Theorem [1.6. Suppose that in Theorem 1.7 we have $J=\Delta_{\sigma}$. Then $Z=C_{G}(A)$ is $\sigma$ - and $\theta$-invariant, and

$$
\mathfrak{z}=(\mathfrak{z} \cap \mathfrak{k}) \oplus(\mathfrak{z} \cap \mathfrak{p} \cap \mathfrak{q}) \oplus(\mathfrak{z} \cap \mathfrak{h}) .
$$

Since $\mathfrak{a}$ is a maximal abelian subspace of $\mathfrak{p} \cap \mathfrak{q}, \mathfrak{z} \cap \mathfrak{p} \cap \mathfrak{q}=\mathfrak{a}$. Hence, decomposition (2.12) becomes

$$
\mathfrak{z}=(\mathfrak{z} \cap \mathfrak{k}) \oplus \mathfrak{a} \oplus(\mathfrak{z} \cap \mathfrak{h}),
$$

and we complete the proof as in Theorem 1.7 .

\section{Well-Roundedness OF SECTORS $S_{T}(\Omega, w)$}

First we need a precise description of the measure on the set

$$
K M_{I}\left(w v_{0}\right) \cong K M_{I} /\left(M_{I} \cap w H w^{-1}\right) .
$$

3.1. Description of a measure on $K M_{I} /\left(M_{I} \cap w H w^{-1}\right)$. Fix $w \in \mathcal{W}$. Let $\sigma_{w}=i_{w} \circ \sigma \circ i_{w}^{-1}$ be the involution as in Remark 2.1. Then $\sigma_{w} \circ \theta=\theta \circ \sigma_{w}$. Also the semisimple group $M_{I}$ is stable under $\sigma_{w}$ and $\theta$, and hence $M_{I}$ admits the generalized Cartan decomposition (see GOS, Proposition 4.22]):

$$
M_{I}=\left(M_{I} \cap K\right) A^{I}\left(M_{I} \cap w H w^{-1}\right)=\left(M_{I} \cap K\right) A^{I,+} \mathcal{W}_{I}\left(M_{I} \cap w H w^{-1}\right),
$$

where $A^{I}$ is the orthogonal complement of $A_{I}$ in $A$ and it is the Cartan subalgebra of $M_{I}$ associated to the symmetric pair $\left(M_{I} \cap K, M_{I} \cap w H w^{-1}\right)$, and $A^{I,+}=\{a \in$ $\left.A^{I}: \alpha(\log a) \geq 0, \forall \alpha \in I\right\}$ is a positive Weyl chamber; and $\mathcal{W}_{I} \subset M_{I}$ is a set of representatives of the associated Weyl group, which is generated by the reflections $\left\{s_{\alpha}\right\}_{\alpha \in I}$. An invariant measure, say $\lambda$ on $M_{I} /\left(M_{I} \cap w H w^{-1}\right)$ is given as follows: for any $f \in C_{c}\left(M_{I} / M_{I} \cap w H w^{-1}\right)$,

$$
\int f d \lambda=\sum_{w_{1} \in \mathcal{W}_{I}} \int_{K \cap M_{I}} d k \int_{A^{I,+}} f\left(k a w_{1}\left(M_{I} \cap w H w^{-1}\right)\right) \delta_{I}(a) d a
$$

where

$$
\delta_{I}(a)=\prod_{\alpha \in \Sigma_{\sigma}^{+} \cap\langle I\rangle}(\sinh \alpha(a))^{l_{\alpha}^{+}}(\cosh (\alpha))^{l_{\alpha}^{-}},
$$

and $l_{\alpha}^{ \pm}$denote the dimensions of the $( \pm 1)$-eigenspaces of $\sigma \theta$ on $\mathfrak{g}^{\alpha}$.

Therefore we can identify

$$
K M_{I} /\left(M_{I} \cap w H w^{-1}\right) \cong K \times A^{I,+} \times \mathcal{W}_{I},
$$

and treat $K M_{I} /\left(M_{I} \cap w H w^{-1}\right)$ as a product measure space.

On the other hand, once we fix a measurable section $s_{1}: K /\left(K \cap M_{I}\right) \rightarrow K$ for the natural quotient map, we can identify $K \times A^{I,+} \times \mathcal{W}_{I}$ with $K /\left(K \cap M_{I}\right) \times$ $M_{I} /\left(M_{I} \times w H w^{-1}\right)$. We consider the measure on $K \times A^{I,+} \times \mathcal{W}_{I}$ such that it corresponds to the product of the invariant measures on the product space $K /\left(K \cap M_{I}\right) \times M_{I} /\left(M_{I} \cap w H w^{-1}\right)$, where the Haar measures on $K$ and $K \cap M_{I}$ 
are normalized. This measure, in view of (3.2), will give rise to the integral $d \bar{m}$ on $K M_{I} /\left(M_{I} \cap w H w^{-1}\right)$ given as follows: for any $f \in C_{c}\left(K M_{I} / M_{I} \cap w H w^{-1}\right)$,

$$
\int f(\bar{m}) d \bar{m}:=\sum_{w_{1} \in \mathcal{W}_{I}} \int_{K} d k \int_{A^{I,+}} f\left(k a w_{1}\left(M_{I} \cap w H w^{-1}\right)\right) \delta_{I}(a) d a .
$$

3.2. Volume estimate for the sectors $S_{T}(\Omega, w)$. Let $\lambda_{\iota}$ denote the highest weight for the irreducible representation $\iota$. We express

$$
\lambda_{\iota}=\sum_{\alpha \in \Delta_{\sigma}} m_{\alpha} \alpha
$$

and the sum of positive roots (with multiplicities)

$$
2 \rho=\sum_{\alpha \in \Delta_{\sigma}} u_{\alpha} \alpha
$$

Let $I \subset \Delta_{\sigma}$. Set

$$
\begin{aligned}
a_{I} & =\max \left\{\frac{u_{\alpha}}{m_{\alpha}}: \alpha \in \Delta_{\sigma}-I\right\}, \\
b_{I} & =\#\left\{\alpha \in \Delta_{\sigma}-I: \frac{u_{\alpha}}{m_{\alpha}}=a_{I}\right\} .
\end{aligned}
$$

Proposition 3.8. For any $w \in \mathcal{W}$ and a bounded measurable set $\Omega \subset K M_{I} /\left(M_{I} \cap\right.$ $\left.w H w^{-1}\right)$ with positive measure and zero boundary measure, there exists $C_{I}(\Omega, w)>$ 0 such that

$$
\operatorname{Vol}\left(S_{T}(\Omega, w)\right) \sim_{T \rightarrow \infty} C_{I}(\Omega, w) \cdot T^{a_{I}}(\log T)^{b_{I}-1} .
$$

Proof. From [HS, Theorem 2.5] (see also [GOS]) one deduces that a $G$-invariant measure on $G / H$ is given by

$$
\int_{G / H} f d \mu=\sum_{w \in \mathcal{W}} \int_{\bar{m} \in K M_{I} /\left(M_{I} \cap w H w^{-1}\right)} \int_{a \in A_{I}^{+}} f(\bar{m} a w H) \xi_{I}(a) d a d \bar{m}, \quad f \in C_{c}(G / H),
$$

where $d a$ denotes a Haar measure on $A_{I}$, and $d \bar{m}$ is described in the paragraph following (3.2), and

$$
\xi_{I}(a)=\prod_{\alpha \in \Sigma_{\sigma}^{+}-\langle I\rangle} \sin (\alpha(\log a))^{l_{\alpha}^{+}} \cos (\alpha(\log a))^{l_{\alpha}^{-}} .
$$

Here $l_{\alpha}^{ \pm}$denote the dimensions of the $( \pm 1)$-eigenspaces of $\sigma \theta$ in $\mathfrak{g}_{\alpha}$. We decompose $\xi_{I}$ as a linear combination of functions $\exp (\chi(a))$ where $\chi$ 's are characters of $A_{I}$. Note that $2 \rho$ is the maximal character in this decomposition. In view of equations (3.4), (3.5) , and (3.6), we define

$$
I_{0}=I \cup\left\{\alpha \in \Delta_{\sigma}-I: \frac{u_{\alpha}}{m_{\alpha}}<a_{I}\right\} .
$$


By the computation using GOS, Theorem 6.1], as done in the proof of [GOS, Theorem 6.4], applied to $\mathfrak{a}_{I}$ in place of $\mathfrak{a}$, there exists a locally finite measure $\eta_{I, w}$ on $W$ such that for every $f \in C_{c}(W)$,

$$
\lim _{T \rightarrow \infty} \frac{1}{T^{a_{I}}(\log T)^{b_{I}-1}} \int_{a \in A_{I}^{+}} f\left(a w v_{0} / T\right) \xi_{I}(a) d a=\int_{W} f d \eta_{I, w},
$$

where the measure $\eta_{I, w}$ can be described as follows:

$$
\int_{W} f d \eta_{I, w}=\int_{\bar{b} \in D^{+}} f\left(b\left(w v_{0}\right)^{I_{0}}\right) \tilde{\xi}_{I}(b) d \bar{b}
$$

where $D^{+}=\exp \mathfrak{d}^{+}$,

$$
\mathfrak{d}^{+}=\left\{\bar{b} \in \mathfrak{a}_{I} /\left(\mathfrak{a}_{I_{0}} \cap \operatorname{ker} \rho\right): \alpha(b) \geq 0, \forall \alpha \in I_{0}\right\},
$$

$d \bar{b}$ denotes the Haar measure on $A_{I} /\left(A_{I} \cap \exp (\operatorname{ker} \rho)\right), v_{0}^{I_{0}}$ is the projection of $v_{0}$ to the sum of the weight spaces with weights of the form $\lambda_{\iota}-\sum_{\alpha \in I_{0}} m_{\alpha} \alpha$, $m_{\alpha} \geq 0$, and

$$
\tilde{\xi}_{I}(b)=\left(\prod_{\alpha \in\left(\Sigma_{\sigma}^{+} \cap\left\langle I_{0}\right\rangle\right)-\langle I\rangle} \sin (\alpha(\log b))^{l_{\alpha}^{+}} \cos (\alpha(\log b))^{l_{\alpha}^{-}}\right) \cdot \exp \left(\sum_{\alpha \in \Sigma_{\sigma}^{+}-\left\langle I_{0}\right\rangle} u_{\alpha} \alpha(\log b)\right) \text {. }
$$

Moreover it follows from (3.11) that $\eta_{I, w}$ is a homogeneous measure of degree $a_{I}$.

Fix any $m \in K M$. Let $c>1$ and take a continuous function $\psi:[0, \infty] \rightarrow[0,1]$ such that $\operatorname{supp}(\psi) \subset[0, c]$ and $\psi=1$ on $[0,1]$. Setting $f(y)=\psi(\|m y\|)$, we have

$$
\int_{A_{I}^{+}} \chi_{B_{T}}\left(m a v_{0}\right) \xi_{I}(a) d a \leq \int_{A_{I}^{+}} f\left(a w v_{0} / T\right) \xi_{I}(a) d a .
$$

Now by (3.11) and (3.14),

$$
\limsup _{T \rightarrow \infty} \frac{1}{T^{a_{I}}(\log T)^{b_{I}-1}} \int_{A_{I}^{+}} \chi_{B_{T}}\left(\operatorname{mawv}_{0}\right) \xi_{I}(a) d a \leq \int_{W} f d \eta_{I, w} \leq c^{a_{I}} \eta_{I, w}\left(m^{-1} B_{1}\right) .
$$

The lower estimate for lim inf is proved similarly.

Hence, taking $c \rightarrow 1^{+}$, we obtain

$$
\lim _{T \rightarrow \infty} \frac{1}{T^{a_{I}}(\log T)^{b_{I}-1}} \int_{A_{I}^{+}} \chi_{B_{T}}\left(\operatorname{mawv}_{0}\right) \xi_{I}(a) d a=\eta_{I, w}\left(m^{-1} B_{1}\right) .
$$

In view of (3.2) let $s: K M_{I} /\left(M_{I} \cap w H w^{-1}\right) \rightarrow K M_{I}$ denote the measurable section of the obvious quotient map. Since

$$
\begin{gathered}
S_{T}(\Omega, w)=\Omega A_{I}^{+} w v_{0} \cap B_{T} \\
\operatorname{Vol}\left(S_{T}(\Omega, w)\right)=\int_{\bar{m} \in \Omega} \int_{a \in A_{I}^{+}} \chi_{B_{T}}\left(s(\bar{m}) a w v_{0}\right) \xi(a) d a d \bar{m} .
\end{gathered}
$$


Therefore from (3.15), using the dominated convergence theorem, we deduce that

$$
C_{I}(\Omega, w):=\lim _{T \rightarrow \infty} \frac{\operatorname{Vol}\left(S_{T}(\Omega, w)\right)}{T^{a_{I}}(\log T)^{b_{I}-1}}=\int_{\bar{m} \in \Omega} \eta_{I, w}\left(s(\bar{m})^{-1} B_{1}\right) d \bar{m} .
$$

Note that there exists $\delta>0$ such that $s(\bar{m})^{-1} B_{1} \supset B_{\delta}$ for all $\bar{m} \in \Omega$, and because $\eta_{I, w_{0}}$ is homogeneous, $\eta_{I, w}\left(B_{\delta}\right)>0$. Hence $C_{I}(\Omega, w)>0$.

Remark 3.18. The value of the parameter $C_{I}(\Omega, w)$ in the statement of Proposition 3.8 is given by

$$
C_{I}(\Omega, w)=\nu_{I_{0}, w}\left(B_{1} \cap \Omega D^{+}\left(w v_{0}\right)^{I_{0}}\right),
$$

where $\nu_{I_{0}, w}$ is a $G$-invariant measure on the $G$-orbit $G\left(w v_{0}\right)^{I_{0}}$.

This formula can be justified as follows: combining (3.11), (3.12), (3.15), (3.17) and (3.3) we get

$$
\begin{aligned}
C_{I}(\Omega, w) & =\int_{\bar{m} \in \Omega} d \bar{m} \int_{\bar{b} \in D^{+}} \chi_{B_{1}}\left(\bar{m} \bar{b}\left(w v_{0}\right)^{I_{0}}\right) \xi_{I}(b) d \bar{b} \\
& =\int_{k \in K} d k \int_{a \in A^{I,+}} \int_{\bar{b} \in D^{+}} \chi_{\Omega}(k a) \chi_{B_{1}}\left(k a b\left(w v_{0}\right)^{I_{0}}\right) \delta_{I}(a) \tilde{\xi}_{I}(b) d a d \bar{b}
\end{aligned}
$$

where

$$
\delta_{I}(a)=\prod_{\alpha \in\left(\Sigma_{\sigma}^{+} \cap\langle I\rangle\right)} \sin (\alpha(\log a))^{l_{\alpha}^{+}} \cos (\alpha(\log a))^{l_{\alpha}^{-}},
$$

$l_{\alpha}^{ \pm}$are the dimensions of the $( \pm 1)$-eigenspaces of $\sigma \theta$ acting on $\mathfrak{g}^{\alpha}$.

Since

$$
\mathfrak{a}_{I_{0}} \cap \operatorname{ker} \rho=\mathfrak{a}_{I_{0}} \cap \operatorname{ker} \lambda_{\iota},
$$

it follows from [GOS, Theorem 5.1] that the orbit $G\left(w v_{0}\right)^{I_{0}}$ supports a $G$-invariant measure $\nu_{I_{0}}$. Now comparing the formula (3.20) with the formula (5.3) in GOS, Theorem 5.1], we obtain (3.19).

3.2.1. Upper estimate of volume for $(J, c)$-singular elements in $S_{T}(\Omega, w)$. For $c>$ $0, I \subset \Delta_{\sigma}$, and a bounded measurable $\Omega \subset K M_{I}$, we set

$$
V_{I, w}(c)=\left\{\operatorname{mawv}_{0}: m \in \Omega, a \in A_{I}^{+} \text {with } \alpha(\log a) \leq c \text { for some } \alpha \in \Delta_{\sigma}-I\right\} .
$$

Note that this set is the set of $(J, c)$-singular elements for $J=\Delta_{\sigma} \backslash I$.

Proposition 3.22. For small $c>0$ and sufficiently large $T>0$,

$$
\operatorname{Vol}\left(V_{I, w}(c) \cap B_{T}\right) \ll c \cdot T^{a_{I}}(\log T)^{b_{I}-1} .
$$

Proof. For $\alpha \in \Delta_{\sigma}$, set

$$
U_{c}(\alpha)=\left\{a \in A_{I}^{+}: \alpha(\log a) \leq c\right\} .
$$


There exists $\delta>1$ such that $m^{-1} B_{T} \subset B_{\delta T}$ for all $T>0$. By (3.9), this gives the estimate

$$
\operatorname{Vol}\left(V_{I, w}(c) \cap B_{T}\right) \ll \sum_{\alpha \in \Delta_{\sigma}-I} \int_{a \in A_{I}^{+} \cap U_{c}(\alpha):\left\|a v_{0}\right\|<\delta T} \xi_{I}(a) d a
$$

Now we use the volume computation from [GOS] (see the proof of Theorem 6.4 in [GOS] ) to show for every nonnegative $f \in C_{c}(W)$,

$$
\int_{A_{I}^{+} \cap U_{c}(\alpha)} f\left(a v_{0} / T\right) \xi_{I}(a) d a \ll\left(\int_{A_{I}^{+} \cap U_{c}(\alpha)} f\left(a v^{I_{0}}\right) \tilde{\xi}_{I}(a) d a\right) \cdot T^{a_{I}}(\log T)^{b_{I}-1},
$$

where $I \subset I_{0} \subset \Delta_{\sigma}, v^{I_{0}} \in W$ and $\tilde{\xi}_{I} \in C\left(A^{+}\right)$are as defined in section 3.2. By [GOS, Corollary 4.7] the projection of $v_{0}^{I_{0}}$ on the $\lambda_{\iota}$-eigenspace is nonzero, and the map $A^{+} \rightarrow \mathbb{R}: a \mapsto \lambda_{\iota}(a)$ is proper. Therefore the map $A_{I}^{+} \rightarrow W: a \mapsto a v_{0}^{I_{0}}$ is proper. This implies that there exists a compact $L \subset A_{I}^{+}$such that

$$
L \supset\left\{a \in A_{I}^{+}: a v_{0}^{I_{0}} \in \operatorname{supp} f\right\} .
$$

Then

$$
\begin{aligned}
\int_{A_{I}^{+} \cap U_{c}(\alpha)} f\left(a v_{0} / T\right) \xi_{I}(a) d a & \ll \max (f) \cdot \operatorname{Vol}\left(L \cap U_{c}(\alpha)\right) \cdot T^{a_{I}}(\log T)^{b_{I}-1} \\
& \ll_{f} c \cdot T^{a_{I}}(\log T)^{b_{I}-1} .
\end{aligned}
$$

Taking a function $f$ satisfying $\chi_{B_{1}} \leq f$, we obtain

$$
\begin{aligned}
\int_{a \in A_{I}^{+} \cap U_{c}(\alpha):\left\|a v_{0}\right\|<T} \xi_{I}(a) d a & \ll\left(\int_{A_{I}^{+} \cap U_{c}(\alpha)} f(a v) \tilde{\xi}_{I}(a) d a\right) \cdot T^{a_{I}}(\log T)^{b_{I}-1} \\
& \ll_{f} c \cdot T^{a_{I}}(\log T)^{b_{I}-1} .
\end{aligned}
$$

Therefore, by (3.23),

$$
\operatorname{Vol}\left(V_{I, w}(c) \cap B_{T}\right) \ll c \cdot(\delta T)^{a_{I}}(\log (\delta T))^{b_{I}-1} .
$$

This completes the proof.

The following corollary of Theorem 1.7 will be used in the proof of Theorem 1.13 :

Corollary 3.24. Let $\Delta_{\sigma}=I \sqcup J$ and $B$ be a bounded subset of $K M_{I}$. Then given $c>0$, there exist $\ell>1$ and $\varepsilon_{0}>0$ such that for every $(J, c)$-regular $g=b a h \in B A_{I} H$ and $0<\varepsilon<\varepsilon_{0}$,

$$
\mathcal{O}_{\varepsilon} g \subset\left(K \cap \mathcal{O}_{\ell \varepsilon}\right) b\left(M_{I} \cap \mathcal{O}_{\ell \varepsilon}\right)\left(A_{I} \cap \mathcal{O}_{\ell \varepsilon}\right) a H
$$


Proof. Let $b=k m$ for $k \in K$ and $m \in M_{I}$. Note that $m \in K B \cap M_{I}$, which is bounded. By (3.1) there exist $k_{0} \in M_{I} \cap K, a_{0} \in A^{I}$ and $h_{0} \in M_{I} \cap H$ such that $m=k_{0} a_{0} h_{0}$. By Theorem 1.7,

$$
\mathcal{O}_{\varepsilon} g \subset\left(K \cap \mathcal{O}_{\ell \varepsilon}\right) k k_{0}\left(M_{I} \cap \mathcal{O}_{\ell \varepsilon}\right)\left(A_{I} \cap \mathcal{O}_{\ell \varepsilon}\right) a_{0} a H .
$$

There exists $\sigma>1$ such that for every $k \in K$ and small $\varepsilon>0, k \mathcal{O}_{\varepsilon} k^{-1} \subset \mathcal{O}_{\sigma \varepsilon}$. Hence,

$$
\mathcal{O}_{\varepsilon} g \subset\left(K \cap \mathcal{O}_{\ell \varepsilon}\right) k\left(M_{I} \cap \mathcal{O}_{\sigma \ell \varepsilon}\right) k_{0} a_{0} h_{0}\left(A_{I} \cap \mathcal{O}_{\ell \varepsilon}\right) a H
$$

There exists $\eta>1$ such that for every $m \in K B$ and small $\varepsilon>0, m^{-1} \mathcal{O}_{\varepsilon} m \subset \mathcal{O}_{\eta \varepsilon}$. Hence,

$$
\mathcal{O}_{\varepsilon} g \subset\left(K \cap \mathcal{O}_{\ell \varepsilon}\right) k m\left(M_{I} \cap \mathcal{O}_{\eta \sigma \ell \varepsilon}\right)\left(A_{I} \cap \mathcal{O}_{\ell \varepsilon}\right) a H
$$

as required.

Proof of Theorem 1.13. Due to Remark 2.1 without loss of generality, we may assume that $w=e$. We will denote $S_{T}(\Omega, e)$ by $S_{T}(\Omega)$.

Let $c, \varepsilon \in(0,1)$.

Let $s: K M_{I} /\left(M_{I} \cap H\right) \rightarrow K M_{I}$ be a measurable section such that $s(\Omega)$ is bounded and measurable. For neighborhoods $U_{1}$ of $e$ in $K$ and $U_{2}$ of $e$ in $M_{I}$, we set

$$
\begin{aligned}
& \Omega^{+}=U_{1} s(\Omega) U_{2}\left(M_{I} \cap H\right), \\
& \Omega^{-}=\bigcap_{u_{1} \in U_{1}, u_{2} \in U_{2}} u_{1} s(\Omega) u_{2}\left(M_{I} \cap H\right) .
\end{aligned}
$$

One can check that as $U_{1}$ and $U_{2}$ shrink to $\{e\}$, we have

$$
\Omega^{+} \downarrow \bar{\Omega} \quad \text { and } \quad \Omega^{-} \uparrow \operatorname{int}(\Omega) \text {. }
$$

Since $\operatorname{Vol}(\partial \Omega)=0$, we have $\operatorname{Vol}\left(\Omega^{+}-\Omega^{-}\right) \rightarrow 0$. Hence, it follows from (3.17) that we can choose $U_{1}$ and $U_{2}$ so that

$$
C_{I}\left(\Omega^{+}\right)-C_{I}\left(\Omega^{-}\right)<\varepsilon .
$$

Fix a set $\tilde{\Omega} \supset \Omega$ such that $\bar{\Omega} \subset \operatorname{int}(\tilde{\Omega})$, set

$$
V_{I}=\Omega A_{I}^{+} v_{0} \quad \text { and } \quad \tilde{V}_{I}=\tilde{\Omega} A_{I}^{+} v_{0},
$$

and define $V_{I}(c)=V_{I, e}(c)$ and $\tilde{V}_{I}(c)=\tilde{V}_{I, e}(c)$ as in Proposition 3.22. We can choose $U_{1}$ and $U_{2}$ so that $\Omega^{+} \subset \tilde{\Omega}$.

We claim that there exists a neighborhood $\mathcal{O}^{\prime}$ of $e$ in $G$ such that

$$
\mathcal{O}^{\prime} \cdot S_{T}(\Omega) \subset S_{(1+\varepsilon) T}\left(\Omega^{+}\right) \cup\left(\tilde{V}_{I}(c) \cap B_{(1+\varepsilon) T}\right) .
$$

By Corollary 3.24, there exists a neighborhood $\mathcal{O}_{1}$ such that

$$
\mathcal{O}_{1}^{-1} \cdot\left(V_{I}-\tilde{V}_{I}(c)\right) \subset \tilde{V}_{I}-V_{I}(c / 2) .
$$

This implies that

$$
\mathcal{O}_{1} \cdot V_{I}(c / 2) \subset \tilde{V}_{I}(c) .
$$


Also, by Corollary 3.24 and continuity of operator norm, there exists a neighborhood $\mathcal{O}_{2}$ of $e$ in $G$ such that for every $v=\operatorname{mav}_{0} \in V_{I}-V_{I}(c / 2)$,

$$
\mathcal{O}_{2} v \subset\left(U_{1} m U_{2}\right) A_{I}^{+} v_{0}
$$

and

$$
\mathcal{O}_{2} \cdot B_{T} \subset B_{(1+\varepsilon) T}
$$

Hence,

$$
\mathcal{O}_{2} \cdot\left(S_{T}(\Omega)-V_{I}(c / 2)\right) \subset S_{(1+\varepsilon) T}\left(\Omega^{+}\right) .
$$

Setting $\mathcal{O}^{\prime}=\mathcal{O}_{1} \cap \mathcal{O}_{2}$, we deduce the claim (3.26).

Similar argument shows there exists a neighborhood $\mathcal{O}^{\prime \prime}$ of $e$ in $G$ such that

$$
S_{(1-\varepsilon) T}\left(\Omega^{-}\right) \subset\left(\bigcap_{g \in \mathcal{O}^{\prime \prime}} g S_{T}(\Omega)\right) \cup \tilde{V}_{I}(c) .
$$

Combining (3.26) and (3.27), we deduce that for $\mathcal{O}=\mathcal{O}^{\prime} \cap \mathcal{O}^{\prime \prime}$,

$$
\begin{aligned}
& \operatorname{Vol}\left(\mathcal{O} \cdot \partial S_{T}(\Omega)\right) \leq \operatorname{Vol}\left(\mathcal{O} S_{T}(\Omega)-\cap_{g \in \mathcal{O}} g S_{T}(\Omega)\right) \\
& \leq \operatorname{Vol}\left(S_{(1+\varepsilon) T}\left(\Omega^{+}\right)\right)-\operatorname{Vol}\left(S_{(1-\varepsilon) T}\left(\Omega^{-}\right)\right)+\operatorname{Vol}\left(\tilde{V}_{I}(c) \cap B_{(1+\varepsilon) T}\right) .
\end{aligned}
$$

By Proposition 3.22 ,

$$
\limsup _{T \rightarrow \infty} \frac{\operatorname{Vol}\left(\tilde{V}_{I}(c) \cap B_{(1+\varepsilon) T}\right)}{T^{a_{I}}(\log T)^{b_{I}-1}} \ll c .
$$

By Proposition 3.8,

$$
\begin{aligned}
& \lim _{T \rightarrow \infty} \frac{\operatorname{Vol}\left(S_{(1+\varepsilon) T}\left(\Omega^{+}\right)\right)}{T^{a_{I}}(\log T)^{b_{I}-1}}=(1+\varepsilon)^{a_{I}} C_{I}\left(\Omega^{+}\right), \\
& \lim _{T \rightarrow \infty} \frac{\operatorname{Vol}\left(S_{(1-\varepsilon) T}\left(\Omega^{-}\right)\right)}{T^{a_{I}}(\log T)^{b_{I}-1}}=(1-\varepsilon)^{a_{I}} C_{I}\left(\Omega^{-}\right) .
\end{aligned}
$$

Hence, it follows from (3.28) and (3.25) that

$$
\begin{aligned}
\limsup _{T \rightarrow \infty} \frac{\operatorname{Vol}\left(\mathcal{O} \cdot \partial S_{T}(\Omega)\right)}{T^{a_{I}}(\log T)^{b_{I}-1}} & \ll(1+\varepsilon)^{a_{I}} C_{I}\left(\Omega^{+}\right)-(1-\varepsilon)^{a_{I}} C_{I}\left(\Omega^{-}\right)+c \\
& \ll \varepsilon+c .
\end{aligned}
$$

Since $\varepsilon$ and $c$ can be taken arbitrary small, this proves that the family of sets $S_{T}(\Omega)$ is well-rounded. Hence, it follows from [DRS, EM] that

$$
\#\left(\Gamma v_{0} \cap S_{T}(\Omega)\right) \sim_{T \rightarrow \infty} \operatorname{Vol}\left(S_{T}(\Omega)\right) .
$$

This proves the theorem. 
Proof of Theorem 1.4. To deduce Theorem 1.4 from Theorem 1.13, we observe that (see [GOS, §2.3]):

$$
\mathcal{Q}_{W} \simeq \bigcup_{p+q=d} \mathrm{SL}_{d}(\mathbb{R}) / \mathrm{SO}(p, q), \quad d=\operatorname{dim} W
$$

and $\mathrm{SL}_{d}(\mathbb{R}) / \mathrm{SO}(p, q)$ is an affine symmetric space. We set

$$
\begin{aligned}
G & =\mathrm{SL}_{d}(\mathbb{R}), \\
K & =\mathrm{SO}(d), \\
A & =\left\{\operatorname{diag}\left(s_{1}, \ldots, s_{d}\right): s_{i} \in \mathbb{R}^{+}, s_{1} \cdots s_{d}=1\right\}, \\
H & =\mathrm{SO}(p, q) .
\end{aligned}
$$

Then we have the generalized Cartan decomposition $G=K A H$. The set of simple roots is

$$
\begin{gathered}
\Delta_{\sigma}=\left\{\alpha_{i}(s)=s_{i} s_{i+1}^{-1}: i=1, \ldots, d-1\right\}, \quad \text { and } \\
A^{+}=\left\{\operatorname{diag}\left(s_{1}, \ldots, s_{d}\right): s_{1}>\cdots>s_{d}>0\right\} .
\end{gathered}
$$

In view of (1.2) and (1.3), set

$$
i_{k}=\sum_{i=1}^{k} \operatorname{dim} W_{i}, \quad 1 \leq k \leq n .
$$

Let $I=\Delta_{\sigma} \backslash\left\{\alpha_{i_{1}}, \ldots, \alpha_{i_{n}}\right\}$. Then

$$
M_{I} \simeq \mathrm{SL}_{i_{1}}(\mathbb{R}) \times \mathrm{SL}_{i_{2}-i_{1}}(\mathbb{R}) \times \cdots \times \mathrm{SL}_{d-i_{n}}(\mathbb{R}),
$$

and $A_{I}$ is the centralizer of $M_{I}$ in $A$.

Since the set of integral quadratic forms in the question is a finite union of $\mathrm{SL}_{d}(\mathbb{Z})$-orbits, we conclude that the proof of the theorem reduces to the computation of the asymptotics of $\#\left(\mathrm{SL}_{d}(\mathbb{Z}) \boldsymbol{q}_{0} \cap S_{T}\left(\Omega \Omega^{\prime}\right)\right)$ where $\boldsymbol{q}_{0} \in \mathcal{Q}_{W}(\mathbb{Z})$. This shows that Theorem 1.4 is a particular case of Theorem 1.13, it may be noted that since $d \geq 3$ the subgroups $\mathrm{SO}(p, q)$ are semisimple and $\mathrm{SO}(p, q) \cap \mathrm{SL}-d(\mathbb{Z})$ is a lattice in $\mathrm{SO}(p, q)$.

It remains to compute the parameters $a_{I}$ and $b_{I}$, which are determined by the volume asymptotics in Proposition 3.8 .

If we restrict the character $2 \rho$, which is the sum of all roots in $\Sigma_{\sigma}^{+}$, then we get

$$
\left.\rho\right|_{\operatorname{Lie}\left(A_{I}\right)}=\sum_{k=1}^{n} u_{i_{k}} \alpha_{i_{k}}, \quad \text { where } u_{i_{k}}=i_{k}\left(d-i_{k}\right) .
$$

The highest weight, say $\lambda_{\iota}$, of the representation of $\mathrm{SL}_{d}(\mathbb{R})$ on the space of quadratic forms restricted to $\operatorname{Lie}\left(A_{I}\right)$ is

$$
\left.\lambda_{\iota}\right|_{\operatorname{Lie}\left(A_{I}\right)}=\sum_{k=1}^{n} m_{i_{k}} \alpha_{i_{k}} \quad \text { where } m_{i}=2\left(d-i_{k}\right) / d
$$


By (3.6) and (3.7),

$$
\begin{aligned}
& a_{I}=\max \left\{\frac{u_{i_{k}}}{m_{i_{k}}}: 1 \leq k \leq n\right\}=d i_{n} / 2, \\
& b_{I}=\#\left\{i_{k}: 1 \leq k \leq n, \frac{u_{i_{k}}}{m_{i_{k}}}=a_{I}\right\}=1 .
\end{aligned}
$$

This proves the theorem.

\section{Another Version of the Strong WAVEFront LEMma}

In this section, we obtain a version of the strong wavefront lemma for a generalized Cartan decomposition with a different Weyl chamber $\tilde{A}^{+}$defined below.

Let $G^{\sigma \theta}=\{g \in G: \sigma \theta(g)=g\}$, the symmetric subgroup associated to the involution $\sigma \theta$ of $G$ and $\mathfrak{g}^{\sigma \theta}$ be the associated Lie subalgebra. Then $A$ is the maximal $\mathbb{R}$-split Cartan subalgebra of $G^{\sigma \theta}$. Set

$$
\tilde{\Sigma}_{\sigma, \theta}=\left\{\alpha \in \Sigma_{\sigma}: \mathfrak{g}^{\alpha} \cap \mathfrak{g}^{\sigma \theta} \neq\{0\}\right\} \quad \text { and } \quad \tilde{\Sigma}_{\sigma, \theta}^{+}=\Sigma_{\sigma}^{+} \cap \tilde{\Sigma}_{\sigma, \theta} .
$$

Then $\tilde{\Sigma}_{\sigma, \theta}$ is a root system on $A$, and we denote by $\tilde{\Delta}_{\sigma, \theta} \subset \tilde{\Sigma}_{\sigma, \theta}^{+}$the set of simple roots on $A$. Let $\tilde{A^{+}}$denote the associated closed Weyl chamber of $A$. Then $A^{+} \subset \tilde{A^{+}}$. Also the following generalized Cartan decomposition holds:

$$
G=K \tilde{A}^{+} H .
$$

Note that $\tilde{A}^{+} \neq A^{+}$in general (see [HS, p. 109]).

Given $c>0$, an element $g=k a h \in K \tilde{A^{+}} H$ is called $c$-regular for $\tilde{\Delta}_{\sigma, \theta}$ if $\alpha(a)>c$ for all $\alpha \in \tilde{\Delta}_{\sigma, \theta}$.

Theorem 4.1 (Strong wavefront Lemma-III). Given $c>0$, there exist $\ell>1$ and $\varepsilon_{0}>0$ such that for every $g=k a h \in K \tilde{A^{+}} H$ which is c-regular for $\tilde{\Delta}_{\sigma, \theta}$ and every $0<\varepsilon<\varepsilon_{0}$,

$$
\mathcal{O}_{\varepsilon} g \subset\left(K \cap \mathcal{O}_{\ell \varepsilon}\right) k \cdot\left(A \cap \mathcal{O}_{\ell \varepsilon}\right) a \cdot\left(H \cap \mathcal{O}_{\ell \varepsilon}\right) h .
$$

This result is stronger than Theorem 1.6 because any $c$-regular element is also $c$-regular for $\tilde{\Delta}_{\sigma, \theta}$, but the converse implication does not hold in general.

Now we consider the situation involving singular elements. Let $\tilde{I} \subset \tilde{\Delta}_{\sigma, \theta}$ and $\tilde{J}=\tilde{\Delta}_{\sigma, \theta} \backslash \tilde{I}$. For $c>0$, we say that an element $g=k a h \in K A^{+} H$ is $(\tilde{J}, c)$-regular if $\alpha(\log a)>c$ for all $\alpha \in \tilde{J}$. Let $A_{\tilde{I}}=\exp (\operatorname{ker} \tilde{I})$. Let $M_{\tilde{I}}^{\sigma \theta}$ denote the analytic semisimple subgroup of $G^{\sigma \theta}$ whose Lie algebra is generated by $\mathfrak{g}^{ \pm \beta} \cap \mathfrak{g}^{\sigma \theta}$ for all $\beta \in \Sigma_{\sigma, \theta}^{+} \cap\langle\tilde{I}\rangle$. Then $M_{\tilde{I}}^{\sigma \theta}$ is contained in the centralizer of $A_{\tilde{I}}$, and

$$
G=K M_{\tilde{I}}^{\sigma \theta} A_{\tilde{I}}^{+} H
$$

where $A_{\tilde{I}}^{+}=\tilde{A^{+}} \cap A_{\tilde{I}}$. 
Theorem 4.2 (Strong wave front lemma-IV). Given $c>0$, there exist $\ell>1$ and $\varepsilon_{0}>0$ such that for every $\tilde{I} \subset \tilde{\Delta}_{\sigma \theta}, \tilde{J}=\tilde{\Delta}_{\sigma, \theta} \backslash \tilde{I}, g=k a h \in K \tilde{A}^{+} H$ which is $(\tilde{J}, c)$-regular, and $0<\varepsilon<\varepsilon_{0}$,

$$
\mathcal{O}_{\varepsilon} \cdot g \subset\left(K \cap \mathcal{O}_{\ell \varepsilon}\right) k \cdot\left(M_{\tilde{I}}^{\sigma \theta} \cap \mathcal{O}_{\ell \varepsilon}\right) \cdot\left(A_{\tilde{I}} \cap \mathcal{O}_{\ell \varepsilon}\right) a \cdot\left(H \cap \mathcal{O}_{\ell \varepsilon}\right) h
$$

This result strengthens Theorem [1.7.

Lemma 4.3. For any $a \in A$,

$$
\mathfrak{g}=\mathfrak{q} \oplus(\mathfrak{k} \cap \mathfrak{h}) \oplus \operatorname{Ad} a(\mathfrak{p} \cap \mathfrak{h}) .
$$

Proof. Since $\mathfrak{g}=\mathfrak{q} \oplus(\mathfrak{k} \cap \mathfrak{h}) \oplus(\mathfrak{p} \cap \mathfrak{h})$, it is enough to to show that

$$
\operatorname{Ad} a(\mathfrak{p} \cap \mathfrak{h}) \cap(\mathfrak{q}+(\mathfrak{k} \cap \mathfrak{h}))=\{0\} .
$$

To prove this, let $X \in \mathfrak{p} \cap \mathfrak{h}$ such that $\operatorname{Ad} a(X) \in \mathfrak{q} \oplus(\mathfrak{k} \cap \mathfrak{h})$. Therefore,

$$
\begin{aligned}
& \sigma(\operatorname{Ad} a(X))=\operatorname{Ad} \sigma(a)(\sigma(X))=(\operatorname{Ad} a)^{-1}(X), \\
& \theta(\operatorname{Ad} a(X))=\operatorname{Ad} \theta(a)(\theta(X))=(\operatorname{Ad} a)^{-1}(-X),
\end{aligned}
$$

and

$$
\sigma(\operatorname{Ad} a(X))=-\theta(\operatorname{Ad} a(X))=(\operatorname{Ad} a)^{-1}(X) .
$$

Now we write $\operatorname{Ad} a(X)=Y_{1}+Y_{2}+Y_{3}$, where $Y_{1} \in \mathfrak{q} \cap \mathfrak{k}, Y_{2} \in \mathfrak{q} \cap \mathfrak{p}$, and $Y_{3} \in \mathfrak{k} \cap \mathfrak{h}$. Then

$$
\begin{aligned}
\sigma(\operatorname{Ad} a(X)) & =-Y_{1}-Y_{2}+Y_{3}, \\
\theta(\operatorname{Ad} a(X)) & =Y_{1}-Y_{2}+Y_{3},
\end{aligned}
$$

and it follows from (4.4) that $Y_{2}=0$ and $Y_{3}=0$. Hence, $\operatorname{Ad} a(X) \in \mathfrak{k} \cap \mathfrak{q}$ and $\sigma(\operatorname{Ad} a(X))=-\operatorname{Ad} a(X)$. Then by (4.4),

$$
(\operatorname{Ad} a)^{2}(X)=-X \text {. }
$$

If $X \neq 0$, this gives a contradiction because $\operatorname{Ad} a$ is self-adjoint.

As a consequence of the above lemma, we obtain the following:

Corollary 4.5. Given $c>0$ there exist $\ell>1$ and $\varepsilon_{0}>0$ such that for any $a \in A$ such that $|\alpha(\log a)| \leq c$ for all $\alpha \in \Delta_{\sigma}$, and any $0<\varepsilon<\varepsilon_{0}$, we have

$$
\mathcal{O}_{\varepsilon} a \subset\left(\mathcal{O}_{\ell \varepsilon} \cap K\right)\left(\mathcal{O}_{\ell \varepsilon} \cap \exp (\mathfrak{p} \cap \mathfrak{q})\right) a\left(\mathcal{O}_{\ell \varepsilon} \cap \exp (\mathfrak{p} \cap \mathfrak{h})\right) .
$$

Proof of Theorem 4.2. Let $w \in \mathcal{W}$ be such that $w a w^{-1}=b \in A^{+}$. We set

$$
I=\left\{\alpha \in \Delta_{\sigma}: \alpha(\log b)<c / n_{0}\right\} \quad \text { and } \quad J=\Delta_{\sigma} \backslash I .
$$


where $n_{0} \in \mathbb{N}$ is such that any positive root is a sum of at most $n_{0}$ simple roots counted with multiplicity. We apply Theorem [1.7 to the involution $\sigma_{w}:=$ $i_{w} \circ \sigma \circ i_{w}^{-1}$ in place of $\sigma$. Since the element $\left(k w^{-1}\right) b\left(w h w^{-1}\right)$ is $\left(J, c / n_{0}\right)$-regular,

$$
\begin{aligned}
\mathcal{O}_{\varepsilon}(k a h)= & \mathcal{O}_{\varepsilon}\left(k w^{-1}\right) b\left(w h w^{-1}\right) w \\
\subset & \left(\mathcal{O}_{\ell \varepsilon} \cap K\right)\left(k w^{-1}\right)\left(\mathcal{O}_{\ell \varepsilon} \cap M_{I}\right)\left(\mathcal{O}_{\ell \varepsilon} \cap A_{I}\right) b\left(\mathcal{O}_{\ell \varepsilon} \cap w H w^{-1}\right)\left(w h w^{-1}\right) w \\
= & \left(\mathcal{O}_{\ell \varepsilon} \cap K\right) k\left(w^{-1} \mathcal{O}_{\ell \varepsilon} w \cap w^{-1} M_{I} w\right)\left(w^{-1} \mathcal{O}_{\ell \varepsilon} w \cap w^{-1} A_{I} w\right) a \\
& \times\left(w^{-1} \mathcal{O}_{\ell \varepsilon} w \cap H\right) h .
\end{aligned}
$$

There exists $\ell_{1}>1$ such that

$$
w^{-1} \mathcal{O}_{\ell \varepsilon} w \subset \mathcal{O}_{\ell_{1} \varepsilon}
$$

for all $0<\varepsilon<\varepsilon_{0}$. Since $M_{I}$ is $\sigma_{w^{-}}$and $\theta$-stable, $M_{I}^{w}:=w^{-1} M_{I} w$ is $\sigma$ - and $\theta$-stable and $A=\left(A \cap M_{I}^{w}\right)\left(w^{-1} A_{I} w\right)$. Let $a_{1} \in A \cap M_{I}^{w}$ be such that $a \in a_{1}\left(w^{-1} A_{I} w^{-1}\right)$. We now apply Corollary 4.5 to $M_{I}^{w}$ in place of $G$, and conclude that for some $\ell_{2} \geq \ell_{1}$

$$
\begin{aligned}
\left(\mathcal{O}_{\ell_{1} \varepsilon} \cap M_{I}^{w}\right) a_{1} \subset & \left(\mathcal{O}_{\ell_{2} \varepsilon} \cap K \cap M_{I}^{w}\right)\left(\mathcal{O}_{\ell_{2} \varepsilon} \cap \exp (\mathfrak{p} \cap \mathfrak{q}) \cap M_{I}^{w}\right) a_{1} \\
& \times\left(\mathcal{O}_{\ell_{2} \varepsilon} \cap \exp (\mathfrak{p} \cap \mathfrak{h}) \cap M_{I}^{w}\right) .
\end{aligned}
$$

Since $M_{I}^{w}$ commutes with $w^{-1} A_{I} w$, combining (4.6) and (4.7), we obtain that for some $\ell_{3} \geq \ell_{2}$

$$
\mathcal{O}_{\varepsilon}(k a h) \subset\left(\mathcal{O}_{\ell_{3} \varepsilon} \cap K\right) k\left(\mathcal{O}_{\ell_{3} \varepsilon} \cap \exp (\mathfrak{p} \cap \mathfrak{q}) \cap M_{I}^{w}\right)\left(\mathcal{O}_{\ell_{3} \varepsilon} \cap w^{-1} A_{I} w\right) a\left(\mathcal{O}_{\ell_{3} \varepsilon} \cap H\right) h .
$$

By the definition of $I$, each eigenvalue of $\operatorname{ad}(\log b)$ on the Lie algebra of $M_{I}$ is at most $c$. Hence every eigenvalue of $\operatorname{ad}(\log a)$ on the Lie algebra of $M_{I}^{w}$ is at most $c$. Since $a$ is given to be $(\tilde{J}, c)$-regular, we conclude that

$$
M_{I}^{w} \cap \exp (\mathfrak{p} \cap \mathfrak{q}) \subset M_{I}^{w} \cap G^{\sigma \theta} \subset M_{\tilde{I}} .
$$

Therefore, the conclusion of the theorem follows from (4.8).

Note that Theorem 4.1 follows from Theorem 4.2 .

\section{REFERENCES}

[DRS] W. Duke, Z. Rudnick and P. Sarnak Density of integer points on affine homogeneous varieties, Duke Math. J. 71, 1993, 181-209.

[EM] A. Eskin and C. McMullen Mixing, counting and equidistribution in Lie groups, Duke Math. J. 71, 1993, 143-180.

[EMM] A. Eskin, G. Margulis and S. Mozes Upper bounds and asymptotics in a quantitative version of the Oppenheim conjecture, Ann. of Math. (2) 147 (1998), no. 1, 93-141.

[HS] G. Heckman and H. Schlichtkrull Harmonic analysis and special functions on symmetric spaces, Perspectives in Mathematics, 16. Academic Press, Inc., 1994. 
[GO] A. Gorodnik and H. Oh Orbits of discrete subgroups on a symmetric space and Furstenberg boundary, To appear in Duke Math J.

[GOS] A. Gorodnik, H. Oh and N. Shah Integral points on symmetric varieties and Satake compactifications, Preprint.

[N] A. Nevo Exponential volume growth, maximal functions on symmetric spaces, and ergodic theorems for semi-simple Lie groups, Ergodic Theory Dynam. Systems 25 (2005), no. 4, $1257-1294$.

[Sc] H. Schlichtkrull Hyperfunctions and Harmonic Analysis on Symmetric Spaces, Progress in Mathematics, 49. Birkhaüser Boston, Inc., Boston, MA, 1984.

Mathematics 253-37, Caltech, Pasadena, CA 91106

E-mail address: gorodnik@caltech.edu

Math Department, 151 Thayer St., Brown University, Providence, RI 02912

E-mail address: heeoh@math.brown.edu

School of Mathematics, TIFR, 1 Homi Bhabha Road, Mumbai, 400005, India

E-mail address: nimish@math.tifr.res.in 